\title{
ON FREE PRODUCTS OF GROUPS
}

BY

DANIEL H. WAGNER

1. Introduction. The homomorphisms between two free grsups have been characterized by Herbert Federer and Bjarni Jónsson (see $[\mathbf{F J}]\left({ }^{1}\right)$ ) as being retractive, i.e., if $G$ and $H$ are free groups and if $f$ is a homomorphism on $G$ onto $H$, then $G=S * Z$ with $f$ mapping $S$ isomorphically onto $H$ and $Z$ into the identity. Their proof consists of a transfinite convergence process making strong use of Jakob Nielsen's procedure (see $[\mathbf{N L}]$ or $[\mathbf{F J}]$ ) for reducing a finite subset of a free group. In a footnote, they have expressed belief that their methods could be used to extend a theorem due to I. Gruschko (see [GK] or [K2]) which characterizes the homomorphisms on a finitely generated free group ${ }^{F}$ onto a free product as mapping some free factorization of $F$ onto the given iree factors. It is desired to remove the finitary restriction, the resulting statement amounting to a generalization of the above FedererJónsson result.

With this goal in mind, a procedure is given in $\$ 3$ for reducing a subset of a free product. These reduction transformations are motivated by Nielsen's procedure and possess similar properties. Some important known theorems on the rank and the subgroups of a free product are obtained somewhat as by-products of this development.

The conjecture of Federer and Jónsson is verified in $\$ 4$ (their methods apply quite well). The main result is Theorem 4.4 which can be stated in more familiar notation: if $f$ is a homomorphism on the free group $F$ onto

$$
\prod_{i \in I}^{*} H_{i}
$$

then there exists $J$ such that

$$
F=\prod_{i \in I}^{*} J_{i} \text { and } f\left(J_{i}\right)=H_{i} \text { for } i \in I .
$$

An example is given in 4.5 of an indecomposable group with a decomposable homomorphic image.

In $\$ 5$ some computability assumptions are made within the factors of a free product, and the reduction procedure thereby becomes effective for decision procedure purposes. This fact is applied to prescribe a recursive pro-

Presented to the Society, October 27, 1951 and June 19, 1954; received by the editors April 12, 1954 and, in revised form, April 23, 1956.

(1) We designate references to the Bibliography at the end by symbols in brackets. 
cedure for deciding whether or not a given element of a free product belongs to the subgroup generated by a given finite subset.

This paper is a revised version of the writer's thesis, written under the direction of his esteemed teachers, Professors Jónsson and Federer, and submitted in partial fulfillment of the requirements for the Degree of Doctor of Philosophy at Brown University, April 23, 1951. The revision( $\left.{ }^{2}\right)$ has included simplifying the reduction procedure and the addition of $\$ 5$. The writer is also indebted to Professor H. W. Kuhn for his constructive criticism of this paper.

2. Preliminaries. If $X$ is a set, we denote the cardinal number of $X$ by card $X$. In any numerical inequality, the numbers we compare are integers. When we write $b \in X^{n}$, we mean that $n \geqq 0$ and $b$ is an $n$-termed sequence, $\left\langle b_{1}, \cdots, b_{n}\right\rangle$, with $b_{1}, \cdots, b_{n} \in X$.

Suppose $\mathcal{E}$ is an equivalence relation over the set $X$. For $x, y \in X$, according as $x$ does or does not stand in the relation $\varepsilon$ to $y$, we write

$$
x \notin y \text { or } x \text { not } \& y \text {. }
$$

We designate the 8 -equivalence class of each $x \in X$ by

$$
(x / \varepsilon)=\{y \mid x \& y\} .
$$

In proving Theorem 3.5, we shall compare the fineness of certain equivalence relations over finite sets as measured by

$$
\text { gauge } \mathcal{E}=\prod_{A \in \Delta}(\operatorname{card} A+1) \text {, }
$$

where

$$
\Delta=\{(y / \varepsilon) \mid y \in X\} .
$$

If $G$ is a group, we define [ $A$ ] to be the subgroup of $G$ generated by $A$ for $A \subset G$, and by the rank of $G$ we mean the minimum card $A$ such that $[A]=G$. Thus rank $\{e\}=0$.

The properties of free groups that we use are quite elementary and can be found, for example, in $\$ 2$ of $[\mathrm{FJ}]$.

Suppose $P$ is a subset of the group $G, \tau$ is a function on $P$ into $G$,

$$
C=\{r \mid \tau(r)=r\}
$$

and

$$
\tau(p) \in[C] p^{ \pm 1}[C] \text { for } p \in P .
$$

We then say that $\tau$ is an elementary transformation $\left({ }^{8}\right)$ of $P$. It is clear that

(2) Substantially completed while the writer was on leave from the Operations Evaluation Group, Navy Department. in [FJ].

( $\left.{ }^{2}\right)$ This is a special kind of the elementary transformations of sequential forms considered 
[range $\tau]=[P]$. In fact, if $[P]$ is a free group freely generated by $P$, then range $\tau$ freely generates $[P]$.

Suppose $G$ is a group and $\phi$ is a family of subgroups of $G$ such that

$$
G=\left[\bigcup_{H \in \phi} H\right]
$$

and such that if $K$ is any group and $f_{H}$ is a homomorphism on $H$ into $K$ for $H \in \phi$, then there exists a homomorphism $g$ on $G$ into $K$ such that

$$
g(x)=f_{H}(x) \text { for } \quad x \in H \in \phi .
$$

In this event, $G$ is said to be the free product of the members of $\phi$, in symbols,

$$
G=\prod_{H \in \phi}^{*} H .
$$

If $\phi=\left\{H_{1}, \cdots, H_{k}\right\}$ with $H_{1}, \cdots, H_{k}$ distinct, this is also written

$$
G=H_{1} * \cdots * H_{k} \text {. }
$$

For $H \in \phi, H$ is called a free factor of $G$. We call $\phi$ a free factorization of $G$.

It is clear that if $\phi$ and $\phi^{\prime}$ are respectively free factorizations of the groups $G$ and $G^{\prime}$ and are in one-one correspondence with corresponding factors isomorphic, then $G$ is isomorphic to $G^{\prime}$.

Suppose $\phi$ is a set of groups intersecting pairwise in a common identity $e$. We construct a group $G$ as follows so that $\phi$ is a free factorization of $G$. Let $G$ be the set of finite sequences over

$$
\bigcup_{H \in \phi} H-\{e\},
$$

every pair of consecutive terms of which belong to different members of $\phi$. The product of two elements of $G$ is obtained from the sequence they form in juxtaposition by amalgamating into one term the neighboring end terms if they belong to the same member of $\phi$, deleting the term if the pair cancels, and repeating until neither cancellation nor amalgamation is possible. Identifying $e$ with the vacuous sequence and $u$ with $\langle u\rangle$ whenever $e \neq u \in H \in \phi$, one can check that this construction meets the requirements. (For an easy proof of associativity, see B. L. van der Waerden [WD].)

In view of the preceding two paragraphs, corresponding to each free factorization $\phi$ of a group $G$, each $x \in G$ is uniquely expressible in the form

$$
x=\prod_{i=1}^{n} u_{i}
$$

where $e \neq u_{i} \in H_{i} \in \phi$ for $1 \leqq i \leqq n$ and $H_{i} \neq H_{i+1}$ for $1 \leqq i<n$. Obviously this unique representation property implies the homomorphism property stated 
in the definition of the free product. Another characterization of $\phi$ as a free factorization of $G$ is the property that $\left({ }^{4}\right)$

$$
G=\left[\bigcup_{H \in \phi} H\right] \text { and } \prod_{i=1}^{m} v_{i} \neq e
$$

whenever $m>0, e \neq v_{i} \in H_{i} \in \phi$ for $1 \leqq i \leqq m$, and $H_{i} \neq H_{i+1}$ for $1 \leqq i<m$.

The unique representation described in the preceding paragraph gives rise to a notion of length: $n$ is called the length of $x$ with respect to $\phi$. In particular, $e$ has zero length.

Throughout the remainder of this paper, we shall assume that $\phi$ is a free factorization of the group $G$. Most of our subsequent definitions will be made with respect to $\phi$, but we shall neglect to denote this explicitly.

For $x \in G$, we denote the length of $x$ with respect to $\phi$ by $L(x)$. We fix

$$
\begin{aligned}
& \Sigma=\bigcup_{H \in \in_{\phi}} H=\{u \mid L(u) \leqq 1\}, \\
& \Gamma=\left\{x u x^{-1} \mid u \in \Sigma \text { and } x \in G\right\} .
\end{aligned}
$$

Suppose $x \in G$ and $u$ is the unique member of $\Sigma^{L(x)}$ for which

$$
x=\prod_{i=1}^{L(x)} u_{i}
$$

If $0<\mu \leqq L(x)$, then by $\pi_{\mu}(x)$ we mean the member $H$ of $\phi$ for which $u_{\mu} \in H$, and by $I_{\mu}(x)$ we mean the $\mu$-initial segment of $x$, i.e.,

$$
u_{\mu} \in \pi_{\mu}(x) \in \phi \quad \text { and } \quad I_{\mu}(x)=\prod_{i=1}^{\mu} u_{i} .
$$

If $\mu=0$ or $L(x)<\mu$, we agree that

$$
\pi_{\mu}(x)=\{e\} \text { and } I_{\mu}(x)=e .
$$

We follow [K1] and [NM] in defining, for $x \in G$,

$$
\begin{aligned}
s(x) & =I_{\mu}(x), \\
t(x) & =I_{\mu}\left(x^{-1}\right)^{-1}, \\
c(x) & =s(x)^{-1} x t(x)^{-1},
\end{aligned}
$$

where $\mu$ is $L(x) / 2$ or $(L(x)-1) / 2$ according as $L(x)$ is even or odd. Thus

$$
\Gamma=\left\{x \mid s(x)=t(x)^{-1}\right\},
$$

and, for $x \in G, L(x)$ is even if and only if $c(x)=e$.

We find it convenient to fix an equivalence relation $\pi$ over $G-\{e\}$ suggested by the theorem of A. G. Kurosch on subgroups of a free product (see

(4) Free products of groups have usually been defined in this way. 
[K1]). For $x, y \in G-\{e\}$, we say that $x \varkappa y$ if and only if

$$
x=y \quad \text { or } \quad x=y^{-1} \quad \text { or } \quad x, y \in s(x) \pi_{1}(c(x)) s(x)^{-1} .
$$

If $b \in G^{n}$ and there exist no $j, k$ with $1 \leqq j \leqq k \leqq n$,

$$
b_{j} \mathfrak{K} b_{j+1} \mathfrak{K} \cdots \mathfrak{K} b_{k} \text {, and } \prod_{i=j}^{k} b_{i}=e,
$$

then we say that $b$ is irredundant. Obviously for $a \in G^{m}$ there exists a (possibly vacuous) subsequence $b$ of $a$ such that $b$ is irredundant and

$$
\prod_{i=1}^{m} a_{i}=\prod_{j} b_{j}
$$

If $b \in G^{n}, b$ is irredundant, and $1 \leqq j \leqq k \leqq n$, then $\left\langle b_{j}, \cdots, b_{k}\right\rangle$ is irredundant.

We conclude this section with some lemmas (which can be proved by straightforward checking) regarding length in products of elements $x, y, z$ of $G$. We shall apply Lemma 2.1 frequently but without reference.

It may help the reader in visualizing statments of this nature to use a graphical description such as that employed by F. W. Levi (see [LV]), i.e., represent $s(x)$ and $t(x)$ as sides of an isosceles triangle or trapezoid with $c(x)$ (if different from $e$ ) at the apex. The reader may also find useful the following summary of Lemma 2.1:

$$
L(y) \text { even }
$$

$L(x)>L(x y)$

more than half cancelled or amalgamated

$L(x)=L(x y) \quad$ exactly half cancelled, no amalgamation

$L(x)<L(x y) \quad$ less than half cancelled

\section{$L(y)$ odd}

center cancelled

center amalgamated

center neither cancelled nor amalgamated

2.1. Lemma. Suppose $L(s(y))=\mu$ and $L(s(y) c(y))=\nu>0$. Then

(i) $L(x)>L(x y)$ if and only if

$$
I_{\nu}\left(x^{-1}\right)=s(y) c(y) \text { and } \pi_{\mu+1}\left(x^{-1}\right)=\pi_{\mu+1}(y) ;
$$

(ii) $L(x)=L(x y)$ if and only if

$$
I_{\mu}\left(x^{-1}\right)=s(y)
$$

and

$$
\left(I_{\mu}\left(x^{-1}\right)^{-1} I_{\nu}\left(x^{-1}\right) \neq c(y) \in \pi_{\nu}\left(x^{-1}\right) \quad \text { or } \quad \pi_{\mu+1}\left(x^{-1}\right) \neq \pi_{\mu+1}(y)\right),
$$

which in turn holds if and only if

$$
I_{\mu}\left((x y)^{-1}\right)=t(y)^{-1} \text { and } \pi_{\mu+1}\left((x y)^{-1}\right)=\pi_{\mu+1}\left(x^{-1}\right) ;
$$


(iii) $L(x)<L(x y)$ if and only if

$$
I_{\mu}\left(x^{-1}\right) \neq s(y) \text { or } c(y) \notin \pi_{\nu}\left(x^{-1}\right),
$$

which in turn holds if and only if

$$
I_{\nu}\left((x y)^{-1}\right)=t(y)^{-1} c(y)^{-1} \text { and } \pi_{\mu+1}\left((x y)^{-1}\right)=\pi_{\mu+1}\left(y^{-1}\right) .
$$

2.2. Lemma. If $L(x) \leqq L(x y)$ and $L(y)>L(z)$, then

(i) $L(x y)>L(x y z)$ implies $L(y)>L(y z)$;

(ii) $L(x y)=L(x y z)$ implies $L(y)=L(y z)$.

2.3. Lemma. If $L(x) \leqq L(x y) \geqq L(x y z)$, then

(i) $L(y) \geqq L(z)$ implies $L(y) \geqq L(y z)$;

(ii) $L(y) \leqq L(z)$ implies $L(y z) \leqq L(z)$.

2.4. Lemma. If $L(x)=L(x y) \geqq L(y)$ and $L(x y z)<L(z)$, then $L(y z)=L(z)$.

2.5. Lemma. If $L(x)<L(x y)$ and $L(y z) \geqq L(z)$, then

(i) $L(y)<L(y z)$ implies $L(x y)<L(x y z)$;

(ii) $L(y) \leqq L(z)$ implies $L(x y) \leqq L(x y z)$.

2.6. LemmA. If $L(x) \leqq L(x y), L(y) \geqq L(z)$, and $L(y) \geqq L(y z)$, then $L(x y)$ $\geqq L(x y z)$.

2.7. Lemma. If $L(x) \leqq L(x y) \geqq L(y)$ and $x y \in \Gamma$, then $L(x y) \geqq L(x y x)$.

3. A reduction procedure for free products. In this section we develop properties of the reduction procedure and the associated notion of irreducibility stated in Definition 3.1. We shall make strong use in $\$ 4$ of Theorems 3.3, 3.5, and 3.11 which correspond to key properties of Nielsen's reductions discussed below. In $\$ 5$, we shall apply these results again and in addition Theorem 3.17. The latter theorem describes the structure of elements of a subgroup of a free product in terms of irreducible generators. Some known results are provided by Theorems 3.13, 3.15, and 3.20.

Let us digress, at this point, to discuss the relationship of Definition 3.1 to various choices of generators elsewhere in the literature.

Our motivation comes from Nielsen's procedure (as applied in [FJ]) for reducing a finite subset $A$ of a free group in a finite number of steps to a set which freely generates $[A]$. Nielsen's basic methods appear here as reductions of type (i) and (ii). A reduction of type (ii) must be effected by a multiplier not belonging to $\Gamma$, lest it be repeatable indefinitely. For this reason we include type (iii) reductions, and in making up a multiplier to effect a reduction of type (i) in general we add to $x^{\alpha}$, in Definition 3.1(i), appropriate factors from $\Gamma$. Our reductions are illustrated in Example 3.2.

Because of the different notions of length, Nielsen's reductions do not coincide with the present reductions applied to a free group (which is, of course, a free product of infinite cyclic groups). Indeed, in case $G$ is freely 
generated by $X$ with $x, y, z$ distinct members of $X$,

$$
\begin{array}{crl}
\phi=\{[\{w\}] \mid w \in X\}, & \\
P=\left\{x y^{2}, y^{-2} z\right\}, & Q=\left\{x y^{2}, x z\right\}, \\
P^{\prime}=\left\{x^{3} y, y^{-1} x^{-1} z^{2}\right\}, & \text { and } Q^{\prime}=\left\{x^{3} y, x^{2} z^{2}\right\},
\end{array}
$$

then $Q$ is an $X$ reduction of $P$ and $P$ is irreducible with respect to $\phi$ (i.e., in the sense of Definition 3.1), while $Q^{\prime}$ is a $\phi$ reduction of $P^{\prime}$ and $P^{\prime}$ is irreducible with respect to $X$.

Any subsequent mention of reductions or irreducibility refers to Definition 3.1.

From Theorem 3.6 we find that every finitely generated subgroup of $G$ has an irreducible set of generators. This is not true in general for groups which are not finitely generated, because there exist groups which have no independent set of generators. However, it can be shown that each subgroup of $G$ possesses essentially irreducible generators in the sense of Definition 3.14. (We do not use this fact except in proving known results.) For example, one can choose as generators the set $K$ defined by Marshall Hall, Jr. (see [HL]) as those elements not generated by their predecessors in his semialphabetical well-ordering. Applying Lemma 2 of $[\mathbf{H L}]$ one easily checks that each finite subset of $K$ can be made irreducible by eliminating superfluous transforms.

There exist finite irreducible sets which cannot be realized as Hall's set $K$. For example, if $u, v, w, x, y, z$ differ from $e$ and belong to distinct members of $\phi$ with $v^{2} \neq e \neq y^{2}$ and

$$
P=\left\{u v w, u v^{2} x, u y x, z y^{2} x\right\},
$$

then $P$ is such a set as is any $Q$ with $Q \cup Q^{-1}=P \cup P^{-1}, Q \cap Q^{-1}=0$.

H. W. Kuhn's method (see $[\mathbf{K N}]$ ) of choosing generators using a system of coset functions called a Kurosch system does not in general yield an irreducible set. For example, if $\phi$ consists of four groups of order two generated by $x, y, z, w$ respectively, then $\{x y, x z w y\}$ can be obtained by Kuhn's method and is reducible (type (ii)) to $\left\{x y, y^{-1} z w y\right\}$. We believe that it can be shown that any irreducible set can be obtained by suitably modifying Kuhn's methods, it being necessary to permit his fixed index $\alpha_{0}$ to vary from coset to coset for the purpose $\left(^{(5)}\right.$. Evidently Theorem 3.10 is required to prove this $\left({ }^{5}\right)$.

The conditions imposed by Kurosch, B. H. Neumann, and M. Takahasi in their respective choices of generators in [K1], [NM], and [TK] do not insure irreducibility, although they do insure the property of irreducibility given by Theorem 3.11 .

Let us now return to our development.

(5) Professor Kuhn joins in this conjecture. 
3.1. Definition. Suppose $P$ is a subset of $G, q \in[P]$, and $\tau$ is a function on $P$ into $G$. If (i) (a), (ii)(a), or (iii)(a) holds, we say that $P$ is reducible by the multiplier $q$. If furthermore (i)(b), (ii)(b), or (iii)(b) respectively holds, we say that $\tau$ is a reduction transformation of $P$ effected by $q$.

(i) There exist $x, z \in P$ and $\alpha, \gamma= \pm 1$ such that

(a) we have

$$
\begin{gathered}
q \in x^{\alpha}\left[t\left(x^{\alpha}\right)^{-1} \pi_{1}(c(x)) t\left(x^{\alpha}\right) \cap P-\{z\}\right], \\
x \neq z, \quad L(x)=L(q) \leqq L(z), \quad \text { and } \quad L\left(q z^{\gamma}\right)<L(z) ;
\end{gathered}
$$

(b) $\tau(z)=q z^{\gamma}$ and $\tau(p)=p$ for $z \neq p \in P$.

(ii) There exist $x, y, z \in P$ and $\alpha, \beta, \gamma= \pm 1$ such that

(a) we have

$$
\begin{aligned}
& q=y^{\beta}, y \notin \Gamma, \\
& x \neq y \neq z, \quad L(x) \geqq L(y) \leqq L(z), \\
& L(x) \geqq L\left(x^{\alpha} q\right) \text {, and } L\left(q z^{\gamma}\right) \leqq L(z) \text {; }
\end{aligned}
$$

(b) for $p \in P$, if $p=y$ or $L(p)<L(q)$, then $\tau(p)=p$, otherwise

$$
\tau(p)= \begin{cases}q p q^{-1} & \text { if } L(q p) \leqq L(p) \geqq L\left(p q^{-1}\right), \\ q p & \text { if } L(q p) \leqq L(p)<L\left(p q^{-1}\right), \\ p q^{-1} & \text { if } L(q p)>L(p) \geqq L\left(p q^{-1}\right), \\ p & \text { if } L(q p)>L(p)<L\left(p q^{-1}\right) .\end{cases}
$$

(iii) There exist $x, z \in P, \alpha, \gamma= \pm 1$, and $\mu>0$ such that

(a) we have

$$
\begin{aligned}
& L(s(x)) \geqq \mu \leqq L(s(z)), \\
& q \in\left[I_{\mu}\left(z^{\gamma}\right) \pi_{\mu}\left(z^{\gamma}\right) I_{\mu}\left(z^{\gamma}\right)^{-1} \cap P\right]-\{e\}, \text { and } I_{\mu}\left(x^{\alpha}\right)=q I_{\mu}\left(z^{\gamma}\right) ;
\end{aligned}
$$

(b) for $p \in P$, if $L(p) \leqq L(q)$, then $\tau(p)=p$, otherwise

$$
\tau(p)= \begin{cases}q p q^{-1} & \text { if } I_{\mu}(p)=I_{\mu}\left(z^{\gamma}\right)=I_{\mu}\left(p^{-1}\right), \\ q p & \text { if } I_{\mu}(p)=I_{\mu}\left(z^{\gamma}\right) \neq I_{\mu}\left(p^{-1}\right), \\ p q^{-1} & \text { if } I_{\mu}(p) \neq I_{\mu}\left(z^{\gamma}\right)=I_{\mu}\left(p^{-1}\right), \\ p & \text { if } I_{\mu}(p) \neq I_{\mu}\left(z^{\gamma}\right) \neq I_{\mu}\left(p^{-1}\right) .\end{cases}
$$

We shall refer to reducibility and reduction transformations as being of type (i), (ii), or (iii). By an irreducible set we mean a subset of $G$ which is not reducible.

3.2. Example. The need for each of the three types of reduction transformations in Definition 3.1 may be illustrated as follows. Assume that 
$a, b, c, u, v$ belong to distinct members of $\phi$ and have infinite order. Let

$$
\begin{aligned}
& p_{1}=u a v^{-3} b^{-1} a^{-1}, \\
& p_{2}=a b v b^{-1} a^{-1}, \\
& p_{3}=a b v u^{-2} c^{-1}, \\
& p_{4}=c u c^{-1}, \\
& p_{5}=c u v u a, \\
& P=\left\{p_{1}, p_{2}, p_{3}, p_{4}, p_{5}\right\} .
\end{aligned}
$$

Let $\tau_{1}, \tau_{2}$, and $\tau_{3}$ be the mappings which leave $p_{1}, p_{2}, p_{3}$, and $p_{4}$ fixed with

$$
\begin{aligned}
\tau_{1}\left(p_{5}\right) & =p_{4} p_{5}=c u^{2} v u a, \\
\tau_{2}\left(\tau_{1}\left(p_{5}\right)\right) & =p_{3} p_{4} p_{5}=a b v^{2} u a, \\
\tau_{3}\left(\tau_{2}\left(\tau_{1}\left(p_{5}\right)\right)\right) & =p_{1} p_{2} p_{3} p_{4} p_{5}=u a u a .
\end{aligned}
$$

Then $\tau_{1}, \tau_{2}$, and $\tau_{3}$ are respectively reduction transformations of types (iii), (ii), and (i) effected by the respective multipliers $p_{4}, p_{3}$, and $p_{1} p_{2}$, and $\tau_{3}\left(\tau_{2}\left(\tau_{1}(P)\right)\right)$ is irreducible. Each of the three types of reduction is necessary in that the other two do not suffice to obtain the property of irreducibility stated in Theorem 3.11 (with $x=u a u a$ ).

3.3. Theorem. If $P \subset G$ and $\tau$ is a reduction transformation of $P$, then

(i) $\tau$ is an elementary transformation of $P$;

(ii) $L(\tau(p)) \leqq L(p)$ for $p \in P$;

(iii) if $\tau$ is of type (i), then $L(\tau(p))<L(p)$ for some $p \in P$.

3.4. Lemma. If $k \geqq h$ and $j \geqq 0$, then

$$
(h+j+1)(k+1) \geqq(h+1)(j+k+1),
$$

with equality holding only if $h=k$ or $j=0$.

3.5. TheOREM( $\left.{ }^{6}\right)$. A sequence of reduction transformations applied to a finite set must terminate. Precisely, if $P_{0}, \cdots, P_{m}$ are finite subsets of $G, \tau_{i}$ is a reduction transformation of $P_{i}$ and range $\tau_{i}=P_{i+1}$ for $0 \leqq i<m$, card $P_{0}=n$, and $\lambda=\sum_{p \in P_{0}} L(p)$, then

$$
m+1 \leqq(\lambda+1) 2^{4 \lambda \lambda} .
$$

Proof. For $0 \leqq i \leqq m$ and $\rho>0$ we define $Q_{i}^{p}, Q_{i}^{p}$, and $\mathfrak{C}_{i}^{p}$ to be the equivalence relations over $P_{\boldsymbol{i}} \times\{1,-1\}$ such that if $p, r \in P_{\boldsymbol{i}}$ and $\delta, \epsilon= \pm 1$, then

$(p, \delta) Q_{i}^{p}(r, \epsilon)$ if and only if $I_{p}\left(s\left(p^{\delta}\right)=I_{p}\left(s\left(r^{\boldsymbol{c}}\right)\right)\right.$,

$(p, \delta) \mathbb{B}_{i}^{p}(r, \epsilon)$ if and only if $\pi_{p}\left(s\left(p^{\delta}\right) c(p)\right)=\pi_{\rho}\left(s\left(r^{\bullet}\right) c(r)\right)$,

$(p, \delta) \mathfrak{C}_{i}^{\rho}(r, \epsilon)$ if and only if $(p, \delta) Q_{i}^{\rho}(r, \epsilon)$ and $(p, \delta) \otimes_{i}^{\rho+1}(r, \epsilon)$.

(8) We follow the pattern of 3.8(11) and 3.9 of [FJ]. 
Next we define $\Xi$ to be the set of $(2 \lambda+1)$-termed sequences $\xi$ of integers such that

$$
0 \leqq \xi_{1} \leqq \lambda \text { and } 1 \leqq \xi_{i} \leqq 2^{2 n} \text { for } 1<i \leqq 2 \lambda+1 .
$$

We order $\boldsymbol{\Xi}$ lexicographically so that if $\xi, \eta \in \Xi$, then $\xi<\eta$ if and only if there exists $j>0$ such that

$$
\xi_{i}=\eta_{i} \text { for } 1 \leqq i<j \text { and } \xi_{j}<\eta_{j} .
$$

For $0 \leqq i \leqq m$ we define

$$
\xi^{(i)}=\left\langle\sum_{p \in P_{i}} L(p) \text {, gauge } \mathrm{e}_{i}^{\lambda} \text {, gauge } a_{i}^{\lambda} \text {, gauge } \mathrm{e}_{i}^{\lambda-1} \text {, gauge } a_{i}^{\lambda-1}, \cdots,\right.
$$

$$
\text { gauge } \left.\mathfrak{C}_{i}^{1} \text {, gauge } \mathfrak{a}_{i}^{1}\right\rangle \text {. }
$$

As a consequence of the inequality of the geometric and arithmetic means, we note that if card $X=2 n<\infty$ and $\varepsilon$ is an equivalence relation over $X$, then gauge $\varepsilon \leqq 2^{2 n}$. Accordingly, $\xi^{(i)} \in \Xi$ for $0 \leqq i \leqq m$. In order to show that

$$
m+1 \leqq \operatorname{card} \Xi=(\lambda+1) 2^{4 \lambda n},
$$

we assume that $0 \leqq i<m$ and show that $\xi^{(i+1)}<\xi^{(i)}$. This is evident if

$$
\sum_{p \in P_{i}} L\left(\tau_{i}(p)\right)<\sum_{p \in P_{i}} L(p) \text {, i.e., } \xi_{1}^{(i+1)}<\xi_{1}^{(i)} .
$$

By Theorem 3.3 we may therefore assume that $\tau_{i}$ is not of type (i) and

$$
L\left(\tau_{i}(p)\right)=L(p) \text { for } p \in P_{i} \text {, i.e., } \xi_{1}^{(i+1)}=\xi_{1}^{(i)} .
$$

Assuming further that $\tau_{i}$ is effected by the multiplier $q$ and $L(s(q))=\nu$, we complete the proof with the following statements:

(i) gauge $Q_{i}^{\rho} \geqq$ gauge $\alpha_{i+1}^{\rho}$ and gauge $\mathfrak{C}_{i}^{\rho} \geqq$ gauge $\mathfrak{C}_{i+1}^{\rho}$ for $\rho>\nu$;

(ii) if $\tau_{i}$ is of type (iii), then gauge $a_{i}^{\nu+1}>$ gauge $a_{i+1}^{\nu+1}$;

(iii) if $\tau_{i}$ is of type (ii) and $c(q)=e$, then

$$
\text { gauge } a_{i}^{\prime}>\text { gauge } a_{i+1}^{\prime} \text { and gauge } e_{i}^{\prime} \geqq \text { gauge } e_{i+1}^{\prime} \text {; }
$$

(iv) if $\tau_{i}$ is of type (ii) and $c(q) \neq e$, then gauge $\mathfrak{C}_{i}^{\nu}>$ gauge $\mathfrak{C}_{i+1}^{\nu}$.

In proving these statements, we let $x, y, z, \alpha, \beta, \gamma, \mu$ play the same roles as in Definition 3.1(ii) or (iii), according to the type of reduction transformation under consideration.

For $p \in P_{i}$ and $\delta= \pm 1$ we have

$$
s\left(\tau_{i}(p)^{\delta}\right) \neq s\left(p^{\delta}\right)
$$

if and only if either $\tau_{i}$ is of type (iii) with 


$$
I_{\nu+1}\left(s\left(p^{\delta}\right)\right)=I_{\mu}\left(z^{\gamma}\right),
$$

or $\tau_{i}$ is of type (ii) with

$$
p \neq y, \quad t(q)^{-1}=I_{\nu}\left(s\left(p^{\delta}\right)\right), \quad \text { and } \quad c(q) \in \pi_{\nu+1}\left(s\left(p^{\delta}\right) c(p)\right) ;
$$

furthermore, if $s\left(\tau_{i}(p)^{\delta}\right) \neq s\left(p^{\delta}\right)$, then, since $L\left(\tau_{i}(p)\right)=L(p)$,

$$
I_{\nu}\left(s\left(\tau_{i}(p)^{\delta}\right)\right)=s(q),
$$

$L(p)>L(q)$ implies

$$
I_{\nu}\left(s\left(\tau_{i}(p)^{\delta}\right)\right)^{-1} s\left(\tau_{i}(p)^{\delta}\right)=c(q) I_{\nu}\left(s\left(p^{\delta}\right)\right)^{-1} s\left(p^{\delta}\right),
$$

and

$$
\pi_{\rho}\left(s\left(\tau_{i}(p)^{\delta}\right) c\left(\tau_{i}(p)\right)\right)=\pi_{\rho}\left(s\left(p^{\delta}\right) c(p)\right) \text { for } \rho>\nabla .
$$

We thereby obtain, for $p, r \in P_{i}, \delta, \epsilon= \pm 1$, and $\rho>\nu$,

$$
(p, \delta) \otimes_{i}^{p}(r, \epsilon) \text { implies }\left(\tau_{i}(p), \delta\right) B_{i+1}^{p}\left(\tau_{i}(r), \epsilon\right)
$$

and

$$
(p, \delta) \mathfrak{Q}_{i}^{\rho}(r, \epsilon) \text { implies }\left(\tau_{i}(p), \delta\right) \mathfrak{Q}_{i+1}^{p}\left(\tau_{i}(r), \epsilon\right),
$$

whence

$$
(p, \delta) \mathfrak{C}_{i}^{\rho}(\boldsymbol{r}, \epsilon) \text { implies }\left(\tau_{i}(p), \delta\right) \mathfrak{C}_{i+1}^{\rho}\left(\tau_{i}(\boldsymbol{r}), \epsilon\right)
$$

We now can apply Lemma 3.4 (with $h=0$ ) to obtain (i).

To prove (ii), we note that if $\tau_{i}$ is of type (iii), then

$$
(x, \alpha) \text { not } Q_{i}^{\nu+1}(z, \gamma) \text { and }\left(\tau_{i}(x), \alpha\right) Q_{i+1}^{\nu+1}\left(\tau_{i}(z), \gamma\right),
$$

and we apply Lemma 3.4 with

$$
h=0, \quad j=\operatorname{card}\left((x, \alpha) / a_{i}^{\nu+1}\right), \quad \text { and } \quad k=\operatorname{card}\left((z, \gamma) / Q_{i}^{\nu+1}\right) .
$$

To prove (iii), we assume that $\tau_{i}$ is of type (ii) and $c(q)=e$. We then have, for $p, r \in P_{i}$ and $\delta, \epsilon= \pm 1$,

$$
\begin{gathered}
(y,-\beta) \neq(p, \delta) Q_{i}^{\nu}(y,-\beta) \text { implies }\left(\tau_{i}(p), \delta\right) Q_{i+1}^{\nu}\left(\tau_{i}(y), \beta\right), \\
(y,-\beta) \text { not } Q_{i}^{\nu}(p, \delta) Q_{i}^{\nu}(r, \epsilon) \text { implies }\left(\tau_{i}(p), \delta\right) Q_{i+1}^{\nu}\left(\tau_{i}(r), \epsilon\right), \\
\text { and }(y, \beta) \text { not } Q_{i}^{\nu}(y,-\beta) .
\end{gathered}
$$

These statements also hold with $\mathfrak{C}_{i}^{\nu}, \mathfrak{C}_{i+1}^{\nu}$ substituted for $\mathfrak{Q}_{i}^{\nu}, \mathfrak{Q}_{i+1}^{\nu}$ respectively. Letting

$$
h=1, \quad i=\operatorname{card}\left((y,-\beta) / \mathfrak{e}_{i}^{\prime}\right)-1, \quad \text { and } \quad k=\operatorname{card}\left((y, \beta) / \mathfrak{e}_{i}^{\prime}\right),
$$


we apply Lemma 3.4 to obtain gauge $\mathfrak{C}_{i}^{p} \geqq$ gauge $\mathfrak{C}_{t+1}^{p}$. Since also

$$
(y, \beta) \neq(x,-\alpha) a_{i}^{\prime}(y, \beta) \text { and }(y,-\beta) \neq(z, \gamma) a_{i}^{\prime}(y,-\beta),
$$

we obtain gauge $a_{i}^{y}>$ gauge $a_{i+1}^{y}$ by substituting $a_{i}^{p}$ for $\mathfrak{C}_{i}^{y}$ in the preceding choice of $h, j, k$ and applying Lemma 3.4. This proves (iii).

We establish (iv) with a proof similar to the foregoing proof of (iii). This completes the proof.

3.6. Theorem. If $P$ is a finite subset of $G$, then there exists $Q$ such that $[Q]=[P]$, card $Q \leqq \operatorname{card} P$, and $Q$ is irreducible.

Proof. Apply Theorems 3.3(i) and 3.5.

3.7. Lemma. If $P$ is irreducible, $b \in\left(P \cup P^{-1}\right)^{8}, b$ is irredundant,

$$
L\left(b_{1}\right) \geqq L\left(b_{1} b_{2}\right), \quad \text { and } L\left(b_{2} b_{3}\right) \leqq L\left(b_{3}\right),
$$

then $b_{2} \in \Gamma$.

Proof. Since $b$ is irredundant, $b_{1} \neq b_{2}^{-1} \neq b_{3}$. If $b_{1}=b_{2}$ or $b_{2}=b_{3}$, then $L\left(b_{2} b_{2}\right)$ $\leqq L\left(b_{2}\right)$, so $b_{2} \in \Gamma$. In the alternative case, $L\left(b_{1}\right) \geqq L\left(b_{2}\right)$, else $L\left(b_{1} b_{2}\right)<L\left(b_{2}\right)$, whence $P$ would be reducible (type (i)) by the multiplier $b_{1}$; similarly $L\left(b_{2}\right)$ $\leqq L\left(b_{3}\right)$. Hence in this case also $b_{2} \in \Gamma$, else $P$ be reducible (type (ii)) by the multiplier $b_{2}$.

3.8. Lemma. If $P$ is irreducible, $n>1, b \in\left(P \cup P^{-1}\right)^{n}, b$ is irredundant, and

$$
L\left(b_{1}\right)=L\left(\prod_{i=1}^{j} b_{i}\right)=L\left(b_{j}\right) \quad \text { and } \quad b_{j} \in \mathrm{\Gamma} \quad \text { for } 1<j \leqq n-1,
$$

then

$$
L\left(\prod_{i=1}^{n} b_{i}\right) \geqq L\left(b_{n}\right) .
$$

Proof. We deny the conclusion. We have, for $1<j<n$,

$$
t\left(b_{1}\right)=s\left(b_{j}\right)^{-1}=t\left(b_{j}\right) \text { and } \pi_{1}\left(c\left(b_{1}\right)\right)=\pi_{1}\left(c\left(b_{j}\right)\right) .
$$

Thus $P$ is reducible (type (i)) by the multiplier $\prod_{i=1}^{n-1} b_{i}$ unless

$$
L\left(b_{1}\right)>L\left(b_{n}\right) \quad \text { or } \quad b_{n}^{ \pm 1} \in\left\{b_{1}, \cdots, b_{n-1}\right\} .
$$

If $L\left(b_{1}\right)>L\left(b_{n}\right)$, then $L\left(b_{n-1}\right)>L\left(b_{n-1} b_{n}\right)$ by Lemma $2.2(\mathrm{i})$, whence $P$ is reducible (type (i)).

Alternatively, $b_{1} \neq b_{j} \neq b_{1}^{-1}$ for $1<j \leqq n$ and $b_{k}^{ \pm 1}=b_{n}$ for some $k$ with $1<k<n$, since $b$ is irredundant. Again $P$ is reducible (type (i)), in contradiction, in this case by the multiplier 


$$
\left(\prod_{i=2}^{n} b_{i}\right)^{-1}
$$

3.9. Lemma. If $P$ is irreducible, $n>1, b \in\left(P \cup P^{-1}\right)^{n}, b$ is irredundant, and

$$
L\left(b_{1}\right)=L\left(\prod_{i=1}^{j} b_{i}\right) \quad \text { for } 1<j \leqq n-1,
$$

then

(i) $L\left(b_{j-1}\right) \geqq L\left(b_{j}\right)$ for $1<j \leqq n-1$;

(ii) if $L\left(b_{1}\right) \geqq L\left(\prod_{i=1}^{n} b_{i}\right)$, then $L\left(b_{n-1}\right) \geqq L\left(b_{n}\right)$;

(iii) $L\left(\prod_{i=1}^{n} b_{i}\right) \geqq L\left(b_{n}\right)$.

Proof. Denying the conjunction of (i) and (ii), we let

$$
m=\min \left\{j \mid L\left(b_{j}\right)<L\left(b_{j+1}\right)\right\}
$$

and

$$
k=\min \left\{j \mid j \leqq m \text { and } L\left(b_{i}\right)=L\left(b_{m}\right) \text { for } j \leqq i \leqq m\right\} .
$$

By Lemma 2.3

$$
L\left(b_{j-1}\right) \geqq L\left(b_{j-1} b_{j}\right) \quad \text { and } \quad L\left(b_{j} b_{j+1}\right) \leqq L\left(b_{j+1}\right) \quad \text { for } k<j \leqq m .
$$

Therefore $b_{j} \in \Gamma$ for $k<j \leqq m$ by Lemma 3.7. Should $k=1$, we would have

$$
L\left(\prod_{i=1}^{m+1} b_{i}\right) \leqq L\left(b_{1}\right)=L\left(b_{m}\right)<L\left(b_{m+1}\right)
$$

in contradiction to Lemma 3.8. Therefore $k>1$ and by Lemmas 2.3 and 3.7, as above, $b_{k} \in \Gamma$. We have

$$
L\left(b_{k-1}\right)>L\left(b_{k}\right)=\cdots=L\left(b_{m}\right)<L\left(b_{m+1}\right) .
$$

Let $\mu=L\left(s\left(b_{k}\right) c\left(b_{k}\right)\right)$. Then for $k \leqq j \leqq m$, by Lemma 2.2(ii) and induction,

$$
\begin{gathered}
L\left(b_{k-1}\right)=L\left(\prod_{i=k-1}^{j} b_{i}\right), \\
b_{j} \in I_{\mu}\left(b_{k-1}^{-1}\right) \pi_{\mu}\left(b_{k-1}^{-1}\right) I_{\mu}\left(b_{k-1}^{-1}\right)^{-1},
\end{gathered}
$$

and

$$
b_{k} \mathfrak{K} b_{j} .
$$

By Lemma 2.3,

$$
L\left(\prod_{i=k-1}^{m} b_{i}\right) \geqq L\left(\prod_{i=k-1}^{m+1} b_{i}\right) \text { or } L\left(\prod_{i=k-1}^{m+1} b_{i}\right) \leqq L\left(b_{m+1}\right)
$$

in both of these cases, 


$$
I_{\mu}\left(b_{k-1}^{-1}\right)^{-1} \prod_{i=k}^{m} b_{i}=I_{\mu}\left(\left(\prod_{i=k-1}^{m} b_{i}\right)^{-1}\right)^{-1}=I_{\mu}\left(b_{m+1}\right) .
$$

Since $b$ is irredundant,

$$
\prod_{i=k}^{m} b_{i} \neq e .
$$

Thus $P$ is reducible (type (iii)), in contradiction, proving (i) and (ii).

If (iii) is false, then

$$
L\left(\prod_{i=1}^{n} b_{i}\right)<L\left(b_{n}\right)
$$

and, by Lemma 2.4 and induction,

$$
L\left(\prod_{i=j}^{n} b_{i}\right)=L\left(\prod_{i=j+1}^{n} b_{i}\right)=L\left(b_{n}\right) \quad \text { for } 1<j<n .
$$

By (i) and (ii) applied to $\left\langle b_{n}^{-1}, \cdots, b_{1}^{-1}\right\rangle, L\left(b_{j}\right) \leqq L\left(b_{j+1}\right)$ for $1 \leqq j<n$, so that $L\left(b_{1}\right)=L\left(b_{j}\right)$ for $1<j<n$. Moreover, by Lemmas 2.3 and $3.7, b_{j} \in \Gamma$ for $1<j<n$. We have contradicted Lemma 3.8. This proves (iii) and completes the proof. then

3.10. Theorem. If $P$ is irreducible, $b \in\left(P \cup P^{-1}\right)^{n}$, and $b$ is irredundant,

$$
L\left(\prod_{i=h}^{h^{\prime}} b_{i}\right) \leqq L\left(\prod_{i=1}^{n} b_{i}\right) \quad \text { for } 1 \leqq h \leqq h^{\prime} \leqq n .
$$

Proof. The theorem is trivial if $n=1$. We assume that $n>1$ and the theorem is true for smaller $n$. We need only show that

$$
L\left(\prod_{i=1}^{n-1} b_{i}\right) \leqq L\left(\prod_{i=1}^{n} b_{i}\right)
$$

Let

$$
\begin{aligned}
& k=\max \left\{j \mid 1 \leqq j<n \text { and } L\left(\prod_{i=1}^{i-1} b_{i}\right)<L\left(\prod_{i=1}^{j} b_{i}\right)\right\} \\
& m=\max \left\{j \mid k \leqq j<n \text { and } L\left(b_{k}\right)=L\left(\prod_{i=k}^{h} b_{i}\right) \text { for } k \leqq h \leqq j\right\} .
\end{aligned}
$$

Then $1 \leqq k \leqq \dot{m}<n$. We have, using the inductive hypothesis,

$$
L\left(\prod_{i=1}^{k-1} b_{i}\right)<L\left(\prod_{i=1}^{k} b_{i}\right) \leqq L\left(\prod_{i=1}^{m} b_{i}\right)
$$

and 


$$
L\left(b_{k}\right)=L\left(\prod_{i=k}^{j} b_{i}\right) \geqq L\left(b_{j}\right) \quad \text { for } k \leqq j \leqq m ;
$$

thus also, by Lemma 3.9(iii),

$$
L\left(\prod_{i=k}^{m+1} b_{i}\right) \geqq L\left(b_{m+1}\right) .
$$

Suppose $m+1<n$. Then by inductive hypothesis and definition of $m$,

$$
L\left(\prod_{i=k}^{m} b_{i}\right)<L\left(\prod_{i=k}^{m+1} b_{i}\right) .
$$

By Lemma 2.5(i), with $x=\prod_{i=1}^{k-1} b_{i}, y=\prod_{i=k}^{m} b_{i}$, and $z=b_{m+1}$, we have

$$
L\left(\prod_{i=1}^{m} b_{i}\right)<L\left(\prod_{i=1}^{m+1} b_{i}\right)
$$

in contradiction to the definition of $k$. Therefore $m=n-1$.

In case $L\left(b_{k}\right) \leqq L\left(b_{n}\right)$, we apply Lemma 2.5 (ii) with $x, y, z$ as above to obtain

$$
L\left(\prod_{i=1}^{n-1} b_{i}\right) \leqq L\left(\prod_{i=1}^{n} b_{i}\right)
$$

Suppose that $L\left(b_{k}\right)>L\left(b_{n}\right)$ and $L\left(\prod_{i=1}^{n-1} b_{i}\right)>L\left(\prod_{i=1}^{m} b_{i}\right)$. Let

$$
l=\max \left\{j \mid k \leqq j<n \text { and } L\left(b_{j}\right)>L\left(b_{n}\right)\right\} .
$$

Then by Lemma 2.2

$$
L\left(b_{l}\right)=L\left(\prod_{i=l}^{j-1} b_{i}\right)=L\left(\prod_{i=l}^{j} b_{\imath}\right)>L\left(b_{j}\right) \quad \text { for } l<j<n
$$

and

$$
L\left(b_{l}\right)=L\left(\prod_{i=l}^{n-1}\right)>L\left(\prod_{i=l}^{n} b_{i}\right)
$$

Since $L\left(b_{j}\right)=L\left(b_{n}\right)$ for $l<j<n$, by Lemma 2.3(ii), the inductive hypothesis, and induction we have

$$
L\left(\prod_{i=j}^{n} b_{i}\right) \leqq L\left(\prod_{i=j+1}^{n} b_{i}\right)
$$

whence

$$
L\left(\prod_{i=j}^{n} b_{i}\right)=L\left(b_{n}\right) \quad \text { for } l<j<n .
$$


By Lemma 3.9(iii) applied to $\left\langle b_{n}^{-1}, \cdots, b^{-1}\right\rangle$ we thus have, in contradiction,

$$
L\left(b_{l}\right) \leqq L\left(\prod_{i=l}^{n} b_{i}\right)
$$

3.11. Theorem. If $P$ is irreducible and $x \in[P]$, then

$$
x \in[P \cap\{p \mid L(p) \leqq L(x)\}] .
$$

Proof. Choose $b \in\left(P \cup P^{-1}\right)^{n}$ such that $x=\prod_{i=1}^{n} b_{i}$, and $b$ is irredundant. Apply Theorem 3.10.

3.12. Theorem. If $P$ is irreducible and $[P]=G$, then $P \subset \Sigma$.

Proof. By Theorem 3.11 we have $[P \cap \Sigma]=G$. Therefore $P-\Sigma=0$, else $P$ be reducible (type (i)).

3.13. Theorem $\left({ }^{7}\right)$. If rank $G$ is finite, then rank $G=\sum_{H \in{ }_{\phi}}$ rank $H$.

Proof. Apply Theorem 3.6 to obtain an irreducible set $Q$ such that $[Q]=G$ and card $Q=\operatorname{rank} G$. Then $Q \subset \Sigma$ by Theorem 3.12. Therefore

$$
\text { rank } H \leqq \text { card }(Q \cap H) \text { for } H \in \phi,
$$

whence

$$
\sum_{H \in \in_{\phi}} \operatorname{rank} H \leqq \operatorname{card} Q=\operatorname{rank} G .
$$

The reverse inequality is obvious.

3.14. Definition. We say that a subset $Q$ of $G$ is essentially irreducible if $Q$ is reducible only by transformations of type (i) with

$$
q \Re x \Re z \in \Gamma \text { and } q z^{\gamma}=e,
$$

referring to the notation of Definition 3.1(i).

Thus if $R$ is a finite subset of an essentially irreducible set, then therc exists $P \subset R$ such that $[P]=[R], P$ is irreducible, and such that if $x \in R-P$, then

$$
x \in[P \cap \Gamma \cap(x / \mathfrak{K})] .
$$

3.15. TheOREM( $\left.{ }^{8}\right)$. If $Q$ is essentially irreducible and

$$
\psi=\{[(x / \mathcal{K}) \cap Q] \mid x \in Q\},
$$

(?) Proved independently by Gruschko (see [GK]) and B. H. Neumann (see [NM]). By elementary cardinality arguments (see Levi, [LV], or [NM]) this theorem also holds if rank $G$ is infinite.

(8) In view of the sixth paragraph of this section, this theorem provides no less than the sixth published proof of the Kurosch subgroup theorem (we cite [K1], [BL], [TK], [KN], and [HL]), although, except in the finitely generated case, we borrow heavily from a previous proof to obtain essentially irreducible generators. 
[March

then $\psi$ is a free factorization of $[Q]$. Moreover, each member of $\psi$ is either infinite cyclic or conjugate to a subgroup of some member of $\phi$.

Proof. Suppose $n>0, a \in\left(Q \cup Q^{-1}\right)^{n}$, and $a$ is irredundant. To prove that $\psi$ is a free factorization of $[Q]$, we must show that

$$
\prod_{i=1}^{n} a_{i} \neq e \text {. }
$$

Referring to Definition 3.14, we obtain $P \subset Q$ such that $P$ is irreducible and

$$
a_{i} \in P \cup P^{-1} \cup\left[P \cap \Gamma \cap\left(a_{i} / \mathfrak{K}\right)\right] \quad \text { for } 1 \leqq i \leqq n .
$$

We further obtain $m \geqq n$ and $b \in\left(P \cup P^{-1}\right)^{m}$ such that $b$ is irredundant and

$$
\prod_{i=1}^{m} b_{i}=\prod_{i=1}^{n} a_{i} .
$$

The nonvanishing of this product then follows from Theorem 3.10. The remainder of the theorem follows from the fact that only members of $\Gamma$ can have finite order.

3.16. Definition. Suppose $b \in G^{n}$ and $1 \leqq k \leqq n$. We say that $k$ is isolated leftwards $\{$ rightwards $\}$ in $b$ if

$$
\begin{gathered}
L\left(\prod_{i=1}^{k-1} b_{i}\right)<L\left(\prod_{i=1}^{k} b_{i}\right) \text { and } L\left(\prod_{i=k}^{n} b_{i}\right) \geqq L\left(\prod_{i=k+1}^{n} b_{i}\right) \\
\left\{L\left(\prod_{i=1}^{k-1} b_{i}\right) \leqq L\left(\prod_{i=1}^{k} b_{i}\right) \text { and } L\left(\prod_{i=k}^{n} b_{i}\right)>L\left(\prod_{i=k+1}^{n} b_{i}\right)\right\}
\end{gathered}
$$

We say that $k$ is isolated in $b$ if $k$ is isolated leftwards in $b$ or $k$ is isolated rightwards in $b$.

3.17. Theorem. If $P$ is irreducible, $b \in\left(P \cup P^{-1}\right)^{n}, b$ is irredundant, and $1<k \leqq n$, then

(i) 1 is isolated leftwards and $n$ is isolated rightwards in $b$;

(ii) $k$ is not isolated leftwards in $b$ if and only if

$$
L\left(\prod_{i=1}^{k-1} b_{i}\right)=L\left(\prod_{i=1}^{k} b_{i}\right) ;
$$

(iii) if $k$ is not isolated leftwards in $b$, then $L\left(b_{k-1}\right) \geqq L\left(b_{k}\right)$;

(iv) if $1 \leqq m<k$, then $k$ is not isolated leftwards in $b$ if and only if $k-m+1$ is not isolated leftwards in $\left\langle b_{m}, \cdots, b_{n}\right\rangle$; then

(v) if $k$ is not isolated leftwards in $b, m<k$, and $m$ is isolated rightwards in $b$,

$$
L\left(\prod_{i=m}^{k-1} b_{i}\right)>L\left(b_{k}\right)
$$


(vi) if $k$ is not isolated in $b$, then $b_{k} \in \Gamma$.

Proof. Both (i) and (ii) follow from Theorem 3.10.

Suppose $k$ is not isolated leftwards in $b$. Let

$$
l=\max \{j \mid 1 \leqq j<k \text { and } j \text { is isolated leftwards in } b\} .
$$

Then for $l<j \leqq k$ by (ii) and Theorem 3.10

$$
L\left(\prod_{i=1}^{l-1} b_{i}\right)<L\left(\prod_{i=1}^{l} b_{i}\right) \leqq L\left(\prod_{i=1}^{j-1} b_{i}\right)=L\left(\prod_{i=1}^{j} b_{i}\right)
$$

so that

$$
L\left(b_{l}\right)=L\left(\prod_{i=l}^{j-1} b_{i}\right)=L\left(\prod_{i=l}^{l} b_{i}\right),
$$

else we contradict Lemma 2.5(i). Therefore $L\left(b_{k-1}\right) \geqq L\left(b_{k}\right)$ by Lemma 3.9(i), proving (iii).

Assuming $1 \leqq m<k$, we note that if $k$ is not isolated leftwards in $b$ or if $k-m+1$ is not isolated leftwards in $\left\langle b_{m}, \cdots, b_{n}\right\rangle$, then by Theorem 3.10 and (iii)

$$
L\left(\prod_{i=m}^{k-1} b_{i}\right) \geqq L\left(b_{k-1}\right) \geqq L\left(b_{k}\right) .
$$

Consequently we can apply Theorem 3.10 and Lemmas 2.3(i) and 2.6 to prove (iv).

Under the hypothesis of (v), applying (iv) and its dual, $k-m+1$ is not isolated leftwards in $\left\langle b_{m}, \cdots, b_{n}\right\rangle$, and $m$ is isolated rightwards in $\left\langle b_{1}, \cdots, b_{k}\right\rangle$; hence

$$
L\left(\prod_{i=m}^{k-1} b_{i}\right)=L\left(\prod_{i=m}^{k} b_{i}\right)>L\left(\prod_{i=m-1}^{k} b_{i}\right) \geqq L\left(b_{k}\right) .
$$

Finally, if $k$ is not isolated in $b$, then by (iv) and (ii)

$$
L\left(b_{k-1}\right)=L\left(b_{k-1} b_{k}\right) \text { and } L\left(b_{k} b_{k+1}\right)=L\left(\dot{b}_{k+1}\right),
$$

so $b_{k} \in \Gamma$ by Lemma 3.7. This proves (vi) and completes the proof.

3.18. Lemma $\left({ }^{(}\right)$. If $Q$ is essentially irreducible and

$$
w \in[Q] \cap \Gamma,
$$

then there exist an equivalence class $A$ in the $\Re$-partitioning of $Q$ and $d \in[Q]$ such that $A \subset \Gamma$ and $w \in d[A] d^{-1}$.

Proof. Choose $P \subset Q, n>0$, and $b \in\left(P \cup P^{-1}\right)^{n}$ such that $P$ is irreducible, $b$ is irredundant, and

( ${ }^{9}$ This lemma corresponds to Hilfssatz 6 of [ $\mathbf{K}_{1}$ ]. 


$$
w=\prod_{i=1}^{n} b_{i}
$$

Let $\lambda$ be the length of $w$ with respect to the free factorization of $[Q]$ given by Theorem 3.15. If $\lambda=1$, i.e.

$$
b_{1} \mathfrak{K} b_{2} \mathfrak{K} \cdots \mathfrak{K} b_{n},
$$

then $s\left(b_{1}\right)=t\left(b_{n}\right)^{-1}$, whence $b_{1} \in \Gamma$, so we take $A=\left(b_{1} / \Re\right) \cap Q$ and $d=e$. Let us assume that $\lambda>1$ and the lemma holds for smaller $\lambda$.

If $b_{1} \mathscr{K} b_{n}$, we find $m$ such that $1 \leqq m<n$ and

$$
b_{1} \nVdash \cdots \Re b_{m} \text { not } \mathscr{K} b_{m+1} \text {, }
$$

and we apply the inductive hypothesis to

$$
\left(\prod_{i=1}^{m} b_{i}\right)^{-1} w \prod_{i=1}^{m} b_{i}
$$

Suppose $b_{1}$ not $\mathfrak{K} b_{n}$. Then $\left\langle b_{1}, \cdots, b_{n}, b_{1}, \cdots, b_{n}\right\rangle$ is irredundant. By Theorem 3.10 and Lemma 2.7 and its dual

$$
L\left(\prod_{i=1}^{n} b_{i}\right)=L\left(\prod_{i=1}^{n} b_{i} b_{1}\right)=L\left(\prod_{i=2}^{n} b_{i} b_{1}\right) .
$$

Therefore by Theorem 3.17(ii), (iv), (vi), $L\left(b_{n}\right)=L\left(b_{n} b_{1}\right)$ and $b_{1} \in \Gamma$. Dually $b_{n} \in \Gamma$ and $L\left(b_{n} b_{1}\right)=L\left(b_{1}\right)$. Therefore $b_{1} \mathscr{K} b_{n}$, in contradiction.

3.19. Lemma. If $Q$ is essentially irreducible, $u, v \in Q \cap \Gamma, d \in[Q]$, and $u \mathfrak{K} d v d^{-1}$, then $u \mathfrak{K} v$.

Proof. We choose $P \subset Q, n, m, l>0$,

$$
b \in\left(P \cup P^{-1}\right)^{n}, \quad x \in\left(P \cup P^{-1}\right)^{m}, \quad y \in\left(P \cup P^{-1}\right)^{l}
$$

such that $P$ is irreducible, $b, x, y$ are irredundant,

$$
d=\prod_{i=1}^{n} b_{i}, \quad u=\prod_{i=1}^{m} x_{i}, \quad v=\prod_{i=1}^{l} y_{i},
$$

$u \nVdash x_{i}$ for $i=1, \cdots, m$ and $v \nVdash y_{i}$ for $i=1, \cdots, l$.

It may be assumed that $u$ not $\nVdash b_{1}$ and $b_{n}$ not $\Re v$ for by conjugation we could arrive at this case or obtain the conclusion. Then

$$
\left\langle x_{1}, \cdots, x_{m}, b_{1}, \cdots b_{n}, y_{1}, \cdots y_{l}, b_{n}^{-1}, \cdots, b_{1}^{-1}\right\rangle
$$

is irredundant. Since $u \nVdash d v d^{-1} \in \Gamma$, by Theorem 3.10 we have

$$
L(u) \leqq L\left(u \prod_{i=1}^{n-1} b_{i}\right) \leqq L(u d) \leqq L(u d v) \leqq L\left(u d v b_{n}^{-1}\right) \leqq L\left(u d v d^{-1}\right) \leqq L(u) .
$$


Consequently by Theorem 3.17 (ii), (iii), (iv)

$$
L\left(b_{n}\right) \geqq L(v) \geqq L\left(b_{n}^{-1}\right) \text { and } L(v)=L\left(v b_{n}^{-1}\right) .
$$

Since $v \in \Gamma$, we also have $L\left(b_{n} v\right)=L(v)$. Therefore $b_{n} \in \Gamma$ by Theorem 3.17(ii), (vi). It follows that $b_{n} \Re v$, in contradiction.

3.20. Theorem( $\left.{ }^{10}\right)$. If $Q$ is essentially irreducible, $H \in \phi, x \in G$, and $[Q] \cap x H x^{-1} \neq\{e\}$, then there exists a unique equivalence class $A$ in the $\Re$ partitioning of $Q$ such that

$$
[Q] \cap x H x^{-1}=d[A] d^{-1} \text { for some } d \in[Q] .
$$

Proof. From Lemmas 3.18 and 3.19 we see that there exists at least one $A$ with the required properties. If $A^{\prime}$ is another such set, then $A=A^{\prime}$, else $[A] *\left[A^{\prime}\right]$ be a free factor of $[Q]$ by Theorem 3.15 , with $[A]$ conjugate in $[Q]$ to $\left[A^{\prime}\right]$, which, as observed by Baer and Levi, is impossible.

4. Homomorphisms on a free group onto a free product. This section is devoted chiefly to proving our main result, Theorem 4.4, which removes the finite rank restriction on Gruschko's theorem characterizing the homomorphisms on a free group onto a free product in a simple natural way. The conjecture of Federer and Jónsson in $[\mathrm{FJ}]$ is thereby verified-we generally follow their methods in 4.1,4.2, and 4.3. Example 4.5 prohibits removing the proviso in Theorem 4.4 that $F$ be free.

It may be noted that with Theorems $3.3(\mathrm{i}), 3.5$, and 3.12 available, an elementary proof of Theorem 4.4 is at hand for the case where rank $F$ is finite. Choose a set $X$ which freely generates $F$, carry out a succession of reduction transformations beginning on $f(X)$ until an irreducible set is obtained and carry out corresponding elementary transformations beginning on $X$.

Once Theorems 4.3 and 4.4 are established, there is no difficulty in obtaining the related results of Federer and Jonsson (Theorems 6.3 and 6.4 of $[\mathrm{FJ}]$ ) which inspired this investigation. One easily proves, for this purpose, that if $f$ is a homomorphism on a free group $F$ onto a cyclic group, then there exist $X$ and $x \in X$ such that $F$ is freely generated by $X$ and

$$
f(y)=e \text { for } x \neq y \in X .
$$

4.1. Lemma. If $f$ is a homomorphism on the group $F$ onto $G, P^{\prime}$ is a finite subset of $F, P=f\left(P^{\prime}\right), P$ is reducible, and

$$
p \notin[P-\{p\}] \text { for } p \in P \cap \Sigma,
$$

then there exist $\tau, \tau^{\prime}, Q, Q^{\prime}$ such that, setting $T=[[P] \cap \Sigma]$,

(i) $\tau$ is a reduction transformation of $P$ and $\tau^{\prime}$ is an elementary transformation of $P^{\prime}$;

(10) This theorem provides the improvement of Reinhold Baer and F. W. Levi (see [BL]) to the Kurosch subgroup theorem (see footnote (8)). 
(ii) $Q=$ range $\tau$ and $Q^{\prime}=$ range $\tau^{\prime}$;

(iii) $P^{\prime} \cap f^{-1}(\Sigma) \subset Q^{\prime}$;

(iv) $r \in P^{\prime}$ implies $r \in\left[Q^{\prime} \cap\{d \mid L(f(d)) \leqq L(f(r))\}\right]$;

(v) $r \in P^{\prime}$ and $f(r) \in T$ implies

$$
\begin{aligned}
& r \in\left[Q^{\prime} \cap\{d \mid L(f(d))<L(f(\boldsymbol{r})) \text { or }\right. \\
& \qquad(f(d) \in T \text { and } L(f(d)) \leqq L(f(\boldsymbol{r})))\}] .
\end{aligned}
$$

Proof. First we observe that if $x \in[P]$ and $s(x), t(x) \in T$, then $x \in T$, and if $x \in T$, then $I_{\mu}(x) \in T$ for $\mu \geqq 0$.

We choose $\tau$ to be a reduction transformation of $P$ effected by a multiplier $q$ subject to the following conditions:

Case I. If possible, $q \in T$.

Case II. If Case I does not apply and $\tau$ is of type (ii), then $t(q) \notin T$.

Fixing $C=\{p \mid \tau(p)=p\}$, we choose $b \in\left(C \cup C^{-1}\right)^{n}$ such that

$$
q=\prod_{i=1}^{n} b_{i}, \quad s(q)=s\left(b_{1}\right), \quad t(q)=t\left(b_{1}\right)
$$

and

$$
t\left(b_{1}\right)=s\left(b_{j}\right)^{-1}=t\left(b_{j}\right) \text { for } 1<j \leqq n .
$$

We further choose $b^{\prime} \in\left(P^{\prime} \cup P^{\prime-1}\right)^{n}$ and $q^{\prime} \in\left[P^{\prime}\right]$ such that

$$
f\left(b_{j}^{\prime}\right)=b_{j} \text { for } 1 \leqq j \leqq n \text { and } q^{\prime}=\prod_{i=1}^{n} b_{i}^{\prime} .
$$

For $r \in P^{\prime}$ we define $\tau^{\prime}(r)^{\bullet}$ to be $q^{\prime} r q^{\prime-1}, q^{\prime} r, r q^{\prime-1}$, or $r$ according as $\tau(f(r))^{\bullet}$ is $q f(r) q^{-1}, q f(r), f(r) q^{-1}$, or $f(r)$ with $\epsilon= \pm 1$. We define $Q=$ range $\tau$ and $Q^{\prime}=$ range $\tau^{\prime}$. Then (i) and (ii) are immediate.

Supposing (iii) is false, choose $r \in P^{\prime}$ such that $f(r) \in \Sigma$ and $\tau^{\prime}(r) \neq r$. Then $\tau$ is of type (i) and $\tau(f(r))=e$, whence

$$
f(r) \in[P-\{f(r)\}],
$$

in contradiction to the hypothesis. Therefore (iii) holds.

To check (iv) and (v) we suppose that $r \in P^{\prime}$ and $p=f(r)$. We have

$$
r \in\left[\left\{b_{1}^{\prime}, \cdots, b_{n}^{\prime}, \tau^{\prime}(r)\right\}\right] \text {. }
$$

Clearly $\tau^{\prime}(r) \in Q^{\prime}$ and, for $1 \leqq j \leqq n, b_{j}^{\prime} \in Q^{\prime} \cup Q^{\prime-1}$, since $b_{j} \in C \cup C^{-1}$.

Assuming that $\tau^{\prime}(r) \neq r$, we have $L\left(b_{j}\right) \leqq L(p)$ for $1 \leqq j \leqq n$ and, by Theorem 3.3(ii), $L(\tau(p)) \leqq L(p)$. Therefore (iv) holds.

Assume further that $p \in T$. Then $t(q) \in T$. Therefore if Case II is in effect, $\tau$ is of neither type (ii) nor (iii) and $L(\tau(p))<L(p)$. In any case,

$$
L(\tau(p))<L(p) \text { or } \tau(p) \in T .
$$

Clearly $q \in T$ implies $b_{j} \in T$ for $1 \leqq j \leqq n$. Therefore to complete the proof of 
(v) we assume Case II (whence $\tau$ is of type (i)) and show that $L(q)<L(p)$. Supposing $L(q)=L(p)$, we choose $\delta= \pm 1$ such that

$$
L\left(b_{1}\right)=L(q)=L(p)>L\left(\prod_{i=1}^{n} b_{i} p^{8}\right),
$$

whence, for $1<j \leqq n, s\left(b_{j}\right)=t\left(b_{j}\right)^{-1}=s\left(p^{\delta}\right) \in T$, so that $b_{j} \in T$. Then $b_{1} \notin T$, else Case I would apply, so $b_{1}, b_{1}^{-1} \notin\left\{b_{2}, \cdots, b_{n}\right\}$, and $P$ is reducible (type (i)) by the multiplier

$$
\left(\prod_{i=2}^{n} b_{i} p^{\delta}\right)^{-1} \in T
$$

Thus Case I applies, in contradiction. This completes the proof.

4.2. Lemma. If $f$ is a homomorphism on the group $F$ into $G$ and $W$ is a finite subset of $F$ freely generating $[W]$, then there exists a set $D$ freely generating $[W]$ such that

$$
W \cap f^{-1}(\Sigma) \subset D
$$

and for each $a \in W$ for which $f(a) \in[[f(W)] \cap \Sigma]$ we have

$$
a \in[D \cap\{d \mid f(b) \in \Sigma \text { or } L(f(b))<L(f(a))\}] .
$$

Proof. Letting $Q_{0}^{\prime}=W$ and $Q_{0}=f(W)$, we apply Lemma 4.1 and Theorem 3.5 to obtain $m \geqq 0$ and, for $1 \leqq j \leqq m$, sets $P_{j}, P_{j}^{\prime}, Q_{j}, Q_{j}^{\prime}, E_{j}, E_{j}^{\prime}$ such that $Q_{m}$ is irreducible and, for $1 \leqq j \leqq m$,

$$
\begin{gathered}
Q_{j-1}^{\prime}=P_{j}^{\prime} \cup E_{j}^{\prime}, \quad P_{j}^{\prime} \cap E_{j}^{\prime}=0, \\
f\left(Q_{j}^{\prime}\right)=Q_{j}, \quad f\left(P_{j}^{\prime}\right)=P_{j}, \quad f\left(E_{j}^{\prime}\right)=E_{j} \subset \Sigma, \\
{\left[Q_{j-1}\right]=\left[P_{j}\right], \quad p \notin\left[P_{j}-\{p\}\right] \text { for } p \in P_{j} \cap \Sigma,}
\end{gathered}
$$

and $Q_{j}^{\prime}$ and $Q_{j}$ are related to $P_{j}^{\prime}$ and $P_{j}$ just as $Q^{\prime}$ and $Q$ are related to $P^{\prime}$ and $P$ in Lemma 4.1 .

For $1 \leqq j \leqq m$ we see that

$$
\begin{gathered}
{[W] \text { is freely generated by } Q_{j}^{\prime} \cup \bigcup_{i=1}^{j} E_{i}^{\prime},} \\
W \cap f^{-1}(\Sigma) \subset Q_{j}^{\prime} \cup \cup_{i=1}^{\prime} E_{i}^{\prime}, \quad\left[Q_{0}\right]=\left[Q_{j}\right],
\end{gathered}
$$

and for each $a \in W$ for which $f(a) \in[[f(W)] \cap \Sigma]$ we have

$$
\begin{aligned}
& a \in\left[\left(Q_{j}^{\prime} \cap\{b \mid f(b) \in \Sigma \text { or } L(f(b))<L(f(a)) \text { or }\right.\right. \\
& \left.\left.\left.\qquad\left(f(b) \in\left[\left[Q_{j}\right] \cap \Sigma\right] \text { and } L(f(b)) \leqq L(f(a))\right)\right\}\right) \cup \bigcup_{i=1}^{\prime} E_{i}^{\prime}\right] .
\end{aligned}
$$


Now $\left[Q_{m}\right] \cap \Sigma \subset\left[Q_{m} \cap \Sigma\right]$ by Theorem 3.11. Consequently

$$
Q_{m} \cap\left[\left[Q_{m}\right] \cap \Sigma\right] \subset \Sigma,
$$

else $Q_{m}$ would be reducible (type (i)). We take

$$
D=Q_{m}^{\prime} \cup \bigcup_{i=1}^{m} E_{i}^{\prime} .
$$

4.3. ThEOREM. If the group $F$ is freely generated by $X$ and $f$ is a homomorphism on $F$ onto $G$, then there exists a set $Y$ freely generating $F$ such that

$$
X \cap f^{-1}(\Sigma) \subset Y \text { and } f(Y) \subset \Sigma .
$$

Proof. Let $\Omega$ be the class of all three-termed sequences $A$ such that

$$
\begin{gathered}
A_{1}, A_{2}, A_{3} \text { are disjoint subsets of } F, \\
F \text { is freely generated by } A_{1} \cup A_{2} \cup A_{3}, \\
X \cap f^{-1}(\Sigma) \subset A_{1}, \quad f\left(A_{1}\right) \subset \Sigma, \\
X-f^{-1}(\Sigma) \supset A_{3}, \quad A_{2} \text { is finite, } \\
\quad \text { and }\left[A_{1} \cup A_{2}\right]=\left[X-A_{3}\right] .
\end{gathered}
$$

We observe that $\left\langle X \cap f^{-1}(\Sigma), 0, X-f^{-1}(\Sigma)\right\rangle \in \Omega$.

We partially order $\Omega$ by the relation $\leqq$ in such a way that for $A, B \in \Omega$ we have $A<B$ if and only if

$$
A \neq B, \quad A_{1} \subset B_{1}, \quad A_{8} \supset B_{3},
$$

and $a \in A_{2}$ implies

$$
a \in\left[B_{1} \cup\left\{b \mid b \in B_{2} \text { and } L(f(b))<L(f(a))\right\}\right] .
$$

We can follow the proof of Theorem 6.3 of [FJ] quite closely to show that if $S$ is a nonvacuous simply-ordered subclass of $\Omega$ containing no maximal element, then

$$
\left\langle\bigcup_{A \in S} A_{1}, 0, \bigcap_{A \in S} A_{3}\right\rangle
$$

is an upper bound of $S$ in $\Omega$. Since $\Omega \neq 0$, we can therefore apply the inductive principle known as Zorn's Lemma to obtain a maximal element $A$ of $\Omega$. To complete the proof it suffices to show that $A_{2} \cup A_{3}=0$, for then we can take $Y=A_{1}$.

Supposing $A_{2} \cup A_{3} \neq 0$, we choose finite nonvacuous sets $R$ and $W$ such that

$$
\begin{gathered}
A_{2} \subset R \subset A_{2} \cup A_{3}, \quad R \subset W \subset A_{1} \cup A_{2} \cup A_{3}, \\
f(R) \subset[[f(W)] \cap \Sigma] .
\end{gathered}
$$

Applying Lemma 4.2, we obtain a set $D$ with the properties stated there.

Let $B$ be the three-termed sequence given by 


$$
B_{1}=A_{1} \cup\left(D \cap f^{-1}(\Sigma)\right), \quad B_{2}=D-B_{1}, \quad \text { and } \quad B_{3}=A_{3}-W .
$$

We continue to follow 6.3 of $[\mathrm{FJ}]$ to show successively that $\left[A_{1} \cup W\right]$ $=\left[B_{1} \cup B_{2}\right], B \in \Omega, A \leqq B$, and $A \neq B$, in contradiction to the maximality of $A$.

4.4. ThEOREM. If $f$ is a homomorphism on the free group $F$ onto $G$, then there exists a free factorization $\psi$ of $F$ in one-one correspondence with $\phi$ such that $f(J) \in \phi$ and $f(J)$ corresponds to $J$ for $J \in \psi$.

Proof. Apply Theorem 4.3 to obtain $X$ such that $F$ is freely generated by $X$ and $f(X) \subset \Sigma$. Choose $H_{0} \in \phi$ such that if $\{e\} \in \phi$, then $H_{0}=\{e\}$. Let

$$
\psi=\left\{\left[X \cap f^{-1}(H-\{e\})\right] \mid H_{0} \neq H \in \phi\right\} \cup\left\{\left[X \cap f^{-1}\left(H_{0}\right)\right]\right\} .
$$

4.5. Example. The following example demonstrates that the requirement that $F$ be free may not be deleted from Theorem 4.4.

Consider the case where $\phi$ consists of two groups of order two. Suppose the group $E$ is freely generated by $\{x, y\}$ and $x \neq y$. Let $N$ be the least normal subgroup of $E$ containing $x^{2} y^{-2}$. We assert that in this case:

(i) there exists a homomorphism on $E / N$ onto $G$;

(ii) there exists no proper free factorization of $E / N$.

Let $f$ be a homomorphism on $E$ onto $G$ such that $f(x), f(y) \in \Sigma$. Then (i) follows from the fact that $N C$ kernel $f$.

Assuming $E / N$ can be decomposed, apply Theorem 4.4 (or Gruschko's theorem) to obtain a set $\{z, w\}$ which freely generates $E$ and such that $z N$ and $w N$ generate the free factors. Then $z N$ or $w N$, say $z N$, has finite order, so that either $f(z)=e$ or $L(f(z))$ is odd, which can be shown to be false(11).

5. Decision procedures in free products. In this section we consider the decidability of the questions of reducibility of finite subsets of $G$ and membership in finitely generated subgroups of $G$.

For each $H \in \phi$ we make the following assumptions:

(i) Multiplication and inversion in $H$ are computable.

(ii) A procedure is at hand, given a finite subset $A$ of $H$ and $u \in H$, for deciding whether or not $u \in[A]$ and for producing, in the event of an affirmative answer, $a \in\left(A \cup A^{-1}\right)^{n}$ such that $u=\prod_{i=1}^{n} a_{i}$.

5.1. TheOREM. If $P$ is a finite subset of $G$, then we can decide whether or not $P$ is reducible and, if so, we can carry out a reduction transformation.

Proof. To test for reducibility of type (i), assuming $P \cap P^{-1}=0$, we examine all $x, z \in P \cup P^{-1}$ such that

(11) Details omitted, since the indecomposability of $E / N$ has become obvious from the test given by A. Shenitzer (see [SN]). Professor Kuhn has proposed a classical approach by showing that each element of $E / N$ can be uniquely represented as $(x y)^{m} y^{n} N$ with $m, n$ arbitrary integers, and deducing that $E / N$ has no elements of finite order. To prove uniqueness his proof requires reference to the Schreier representation of a free product with identified subgroups (see [SR]), but unlike the other two proofs does not require Gruschko's theorem. 
$x \neq z, \quad L(x) \leqq L(z), \quad t(x)^{-1}=I_{\mu}(z), \quad$ and $\quad \pi_{\mu+1}\left(x^{-1}\right)=\pi_{\mu+1}(z)$, where $\mu=L(t(x))$, resolving the question into the question of whether or not, in some such case, $c(x)=e$ or

$$
I_{1}\left(I_{\mu}(z)^{-1} z\right) c(x) \in\left[\left\{c(p) \mid p \in t(x)^{-1} \pi_{1}(c(x)) t(x) \cap P-\{z\}\right\}\right],
$$

which can be decided by assumption (ii). We test for reducibility of type (iii) in a similar manner. Testing for reducibility of type (ii) and carrying out reduction transformations of any type are straightforward.

5.2. Theorem. Suppose we are given a finite subset $P$ of $G$ and $x \in G$. Then we can prescribe a procedure for deciding whether or not $x \in[P]$ and for producing, in the event of an affirmative answer, $b \in\left(P \cup P^{-1}\right)^{n}$ such that $x=\prod_{i=1}^{n} b_{i}$.

Proof. In view of Theorems 5.1, 3.3(i), and 3.5, we may just as well assume that $P$ is irreducible. If $L(x)=1$, then the question is decidable by hypothesis and Theorem 3.11. We assume that $L(x)>1$ and that we have prescribed the procedure for smaller values of $L(x)$.

The answer is affirmative if and only if we can find $b \in\left(P \cup P^{-1}\right)^{n}$ such that $b$ is irredundant and $x=\prod_{i=1}^{n} b_{i}$. We shall consider candidates for the roles of $b_{h}$ and $b_{k}$ in such a sequence where

$$
\begin{aligned}
& h=\max \{i \mid i \text { is isolated leftwards in } b\}, \\
& k=\min \{i \mid h \leqq i \text { and } i \text { is isolated rightwards in } b\} ;
\end{aligned}
$$

thus $1 \leqq h \leqq k \leqq n$. Such $b, h, k$ must satisfy the subsequent conditions. From Theorem 3.17 we deduce that

$$
\begin{aligned}
& L\left(b_{h}\right)=L\left(b_{k}\right), L\left(\prod_{i=1}^{h-1} b_{i}\right)<L\left(\prod_{i=1}^{h} b_{i}\right), \\
& L\left(b_{h}\right)=L\left(\prod_{i=h}^{j} b_{i}\right) \geqq L\left(b_{j}\right) \text { for } h<j \leqq n, \\
& L\left(b_{k}\right)=L\left(\prod_{i=k}^{j} b_{i}\right)>L\left(b_{j}\right) \text { for } k<j \leqq n, \\
& L\left(b_{h}\right)=L\left(b_{j}\right) \text { and } b, \in \Gamma \text { for } h<j<k .
\end{aligned}
$$

We therefore see, where $\mu=L\left(s\left(b_{k}\right)\right)$ and $\nu=L\left(s\left(b_{k}\right) c\left(b_{k}\right)\right)$, that

$$
\begin{aligned}
& t\left(b_{h}\right)=s\left(b_{k}\right)^{-1}, \quad \pi_{1}\left(c\left(b_{h}\right)\right)=\pi_{1}\left(c\left(b_{k}\right)\right), \\
& t\left(b_{h}\right)=s\left(b_{j}\right)^{-1}=t\left(b_{j}\right) \text { and } \pi_{1}\left(c\left(b_{h}\right)\right)=\pi_{1}\left(c\left(b_{j}\right)\right) \text { for } h<j<k \text {, } \\
& t\left(b_{k}\right) \prod_{i=k+1}^{n} b_{i}=I_{\mu}\left(x^{-1}\right)^{-1} \\
& \prod_{i=h}^{k} c\left(b_{i}\right)=I_{\nu}\left(x^{-1}\right)^{-1} I_{\mu}\left(x^{-1}\right), \prod_{i=1}^{h-1} b_{i} s\left(b_{k}\right)=x I_{\nu}\left(x^{-1}\right),
\end{aligned}
$$




$$
\pi_{\nu}\left(b_{k}^{-1}\right)=\pi_{\nu}\left(x^{-1}\right) \text {, and } \pi_{\nu}\left(b_{h}\right)=\pi_{\mu+1}\left(x^{-1}\right) .
$$

We now describe the test to which we put each pair $p, r \in P \cup P^{-1}$ as candidates for the respective roles of $b_{k}$ and $b_{k}$ in the preceding paragraph. In view of Theorem 3.10 and the preceding paragraph, we reject the candidates unless

$$
\begin{gathered}
L(p)=L(r) \leqq L(x), \\
t(p)=s(r)^{-1}, \text { and } \pi_{1}(c(p))=\pi_{1}(c(r)) .
\end{gathered}
$$

Letting $\mu=L(s(r))$ and $\nu=L(s(r) c(r))$, assuming this test met, we determine whether or not

$$
\pi_{\nu}\left(r^{-1}\right)=\pi_{\nu}\left(x^{-1}\right), \quad \pi_{\nu}(p)=\pi_{\mu+1}\left(x^{-1}\right),
$$

and

$$
c(p)^{-1} I_{\nu}\left(x^{-1}\right)^{-1} I_{\mu}\left(x^{-1}\right) c(r)^{-1} \in\left[\left\{c(q) \mid s(r) c(r) s(r)^{-1} \nVdash \mathcal{K} q P\right\}\right] ;
$$

if the last of these three fails and $p=r$, we make an alternative test for the possibility that $h=k$ by determining whether or not

$$
c(p)^{-1} I_{\nu}\left(x^{-1}\right)^{-1} I_{\mu}\left(x^{-1}\right)=e .
$$

If negative, we reject the candidates. If affirmative, we find $z \in\left(P \cup P^{-1}\right)^{l}$ such that $z_{1}=p, z_{l}=r$,

$$
t(p)=s\left(z_{i}\right)^{-1}=t\left(z_{i}\right) \text { and } \pi_{1}(c(p))=\pi_{1}\left(c\left(z_{i}\right)\right) \text { for } 1<i<l
$$

and

$$
\prod_{i=1}^{l} c\left(z_{i}\right)=I_{\nu}\left(x^{-1}\right)^{-1} I_{\mu}\left(x^{-1}\right)
$$

Then

$$
s(p)^{-1}\left(\prod_{i=1}^{l} z_{i}\right) t(r)^{-1}=I_{p}\left(x^{-1}\right)^{-1} I_{\mu}\left(x^{-1}\right)
$$

If $\sigma(r)=e$, then $\mu=\nu$,

$$
\pi_{\mu}\left(x^{-1}\right)=\pi_{1}(t(r)), \text { and } \pi_{\nu}(s(p))=\pi_{\nu+1}\left(x^{-1}\right) .
$$

It follows that, whether or not $c(r)=e$,

$$
L\left(I_{\mu}\left(x^{-1}\right) t(r)\right)<L(r) \leqq L(x) \text { and } L\left(s(p) I_{p}\left(x^{-1}\right)^{-1} x^{-1}\right)<L(x),
$$

from which we are equipped by inductive hypothesis to determine whether or not

$$
I_{\mu}\left(x^{-1}\right) t(r) \in[P] \text { and } s(p) I_{\nu}\left(x^{-1}\right)^{-1} x^{-1} \in[P],
$$

which we next proceed to do. If negative, we reject the candidates. If affirmative, we obtain $w \in\left(P \cup P^{-1}\right)^{j}$ and $y \in\left(P \cup P^{-1}\right)^{m}$ such that 


$$
t(r) \prod_{i=1}^{j} w_{i}=I_{\mu}\left(x^{-1}\right)^{-1}
$$

and

$$
\prod_{i=1}^{m} y_{i} s(p)=x I_{\nu}\left(x^{-1}\right)
$$

If no pair meets the foregoing tests, we conclude that the roles of $b_{h}$ and $b_{k}$ cannot be filled and $x \notin[P]$. If one pair meets the test, then while this pair may not fill the roles of $b_{h}$ and $b_{k}$, we nevertheless have

$$
x=\prod_{i=1}^{m} y_{i} \prod_{i=1}^{l} z_{i} \prod_{i=1}^{i} w_{i} \in[P]
$$

with $y, z, w$ as in the preceding paragraph.

\section{BiBLIOGRAPHY}

[BL] R. Baer and F. W. Levi, Freie Produkte und ihre Untergruppen, Compositio Math. vol. 3 (1936) pp. 391-398.

[FJ] H. Federer and B. Jonsson, Some properties of free groups, Trans. Amer. Math. Soc. vol. 68 (1950) pp. 1-27.

[GK] I. Gruschko, Über die Basen eines freien Produktes von Gruppen, Rec. Math. (Mat. Sbornik) N. S. vol. 8(50) (1940) pp. 169-182 (Russian with German summary).

[HL] M. Hall, Jr., Subgroups of free products, Pacific Journal of Mathematics vol. 3 (1953) pp. $115-120$.

[KN] H. W. Kuhn, Subgroup theorems for groups presented by generators and relations, Ann. of Math. vol. 56 (1952) pp. 22-46.

[K1] A. G. Kurosch, Die Untergruppen der freien Produkte von beliebigen Gruppen, Math. Ann. vol. 109 (1934) pp. 647-660.

[K2] — Teoriya Grupp, Moscow-Leningrad, OGIZ, 1944 (Russian).

[LV] F. W. Levi, On the number of generators of a free product and a lemma of Alexander Kurosch, J. Indian Math. Soc. vol. 5 (1941) pp. 149-155.

[NM] B. H. Neumann, On the number of generators of a free product, J. London Math. Soc. vol. 18 (1943) pp. 12-20.

[NL] J. Nielsen, Om Regning med ikke-kommutative Faktorer og dens Anvendelse $i$ Gruppeteorien, Matematisk Tidsskrift (1921) pp. 77-94 (Danish).

[SR] O. Schreier, Die Untergruppen der freien Gruppen, Abhandlungen aus dem Mathematischen Seminar Hamburg Universität vol. 5 (1926) pp. 161-183.

[SN] A. Shenitzer, Decomposition of a group with a single defining relation into a free product, Proc. Amer. Math. Soc. vol. 6 (1955) pp. 273-279.

[TK] M. Takahasi, Bemerkungen ibber den Untergruppensatz in freien Produkte, Proc. Imp. Acad. Tokyo vol. 20 (1944) pp. 589-594.

[WD] B. L. van der Waerden, Free products of groups, Amer. J. Math. vol. 70 (1948) pp. 527-528.

BROWN UNIVERSITY,

Providence, R. I.

Massachusetts Institute of Technology,

Cambridge, Mass. 